\title{
LIPID EMULSIONS IN THE TREATMENT OF ACUTE POISONING IN THE CLINIC OF TOXICOLOGY AT THE NAVAL HOSPITAL-VARNA, MILITARY MEDICAL ACADEMY-SOFIA
}

\author{
Gabriela Kehayova, Snezha Zlateva, Petko Marinov \\ Department of Pharmacology, Toxicology and Pharmacotherapy, Faculty of Pharmacy, \\ Medical University of Varna, Bulgaria
}

\begin{abstract}
INTRODUCTION: Acute drug intoxications are a serious problem worldwide. The damage to the cardiovascular system is severe and sometimes refractory to standard resuscitation therapy. In recent years, interest has increased in the role of lipid emulsions (LEs) as a potential antidote in patients with severe myocardial damage caused by toxic doses of lipophilic drugs.

AIMS: Clinical analysis of the cardioprotective effects of applied LEs at the Clinic of Toxicology at the Naval Hospital-Varna, Military Medical Academy-Sofia, as an antidote for the treatment of some acute poisoning with a cardiotoxic syndrome.

MATERIALS AND METHODS: The subject of the study were 26 patients with acute cardiotoxic lipophilic drug intoxications treated with LEs at the Clinic of Toxicology at the Naval Hospital-Varna in the period 2016-2018. Illnesses and medications administered to patients are derived from the medical documentation.

RESULTS: A three-year study was conducted in patients with acute exogenous intoxications treated with LEs at the Clinic of Toxicology at the Naval Hospital-Varna in regard to their demographics, concomitant diseases and prescribed treatment during their stay. Rapid recovery of patients' vital signs, short hospital stay and lack of adverse effects of LE administration were found.

CONCLUSION: The good clinical results obtained by us will help the implementation of LE in a number of lipophilic intoxications causing toxic cardiovascular damages, including those with hemodynamic instability.
\end{abstract}

Keywords: lipid emulsions, parenteral nutrition, acute intoxications, lipophilic drugs, cardiotoxicity

Address for correspondence:

Gabriela Kehayova

Faculty of Pharmacy

Medical University of Varna

84 Tzar Osvoboditel Blvd

9000 Varna

e-mail:gabi_stier@yahoo.com

Received: November 16, 2018

Accepted: December 20, 2018

\section{INTRODUCTION}

Acute drug intoxications are a serious health problem worldwide. The most commonly overdosed drugs are sedative/hypnotic agents, analgesics, antidepressants, antipsychotics, stimulants, and cardiovascular drugs. Most of these substances do not have a specific antidote and cause serious and sometimes life-threatening neurological and cardiovas- 
Lipid Emulsions in the Treatment of Acute Poisoning in the Clinic of Toxicology at the Naval Hospital-Varna, Military Medical Academy-Sofia

cular complications, requiring the routine introduction of more effective treatments such as intravenous LE. According to Marinov (2018), 6601 patients with acute drug intoxications were hospitalized in the Clinic of Toxicology at the Naval Hospital-Varna for the period 1991 - 2015. The relative share of all poisonings was $37.85 \%$, confirming their leading position in acute intoxications (1).

LEs are a new method that has recently been seen as an alternative to the treatment of poisoning with lipophilic toxic compounds. Reports and scientific publications on LE application in the United States and Europe have been steadily increasing over the past decade, with $85.86 \%$ of the articles being published between 2006 and 2015, and the annual average growth in this area being 51 articles per year. The highest increase has been observed since 2006, when the first report on successful resuscitation with LEs in humans was published (2). Since then, LEs have been extensively used in neuro- and cardiotoxic lipophilic drug intoxications, including local anesthetics, tricyclic antidepressants, and nondihydropyridine calcium channel blockers.

Originally, LEs were designed to satisfy the need for essential fatty acids in patients on parenteral nutrition. Applied as an individual infusion or combined with parenteral nutrition, the fatty acids provided by LEs are vital for cellular structural function and metabolism. Over the last 15 years, a new LE effect has been found, namely their ability to capture lipophilic drugs, and to extract them from vital organs such as the heart and brain, and thus reduce their toxicity (3).

There are many supposed mechanisms of action of LEs as an antidote: the theory of „lipid sink”, the theory of improved metabolism by increasing the content of intracellular fatty acids and overcoming the reduced production of adenosine triphosphate, an increase in calcium ion influx, vasoconstrictor effects. Initially described by Weinberg, the phenomenon of "lipid sinking" is the most common mechanism of action of LEs (3). According to him, LE is found as fat drops or multilayer vesicles in the blood. They bind to the lipophilic toxin and remove it from the target tissue (4). Considering the metabolism of fatty acids, myocardial tissue supplies most of its energy needs from mitochondrial fatty acid oxidation.
Recent studies support the theory that LEs increase the intracellular concentration of fatty acids and delivers additional energy to the myocardium, which in turn increases the levels of calcium and produces a positive inotropic effect $(1,5)$.

In Bulgaria, LEs are not yet the first-line treatment in lipophilic drug intoxications. Shmilev (6), Marinov $(7,8,9)$ and Zlateva (10) report on their treatment options. In Bulgaria there are no studies to prove the cardioprotective effect of LE on both experimental animals and humans.

\section{AIM}

Clinical analysis of the cardioprotective effects of applied LEs in the Clinic of Toxicology at the Naval Hospital-Varna as an antidote for the treatment of some acute poisoning with cardiotoxic syndrome was conducted.

\section{MATERIALS AND METHODS}

The subject of the study were 26 patients with acute intoxications with cardiotoxic lipophilic drugs treated with LE at the Clinic of Toxicology at the Naval Hospital-Varna in the period 2016-2018. The data from the concomitant diseases and the applied drugs during the hospital stay of the patients were obtained from the medical documentation. The following indicators were analyzed: sex, age, comorbidity, drugs used for self-poisoning, cardiovascular complications during the course of intoxication, prescribed treatment, including LE, duration of hospitalization, outcome of treatment. During the hospitalization of the patients the dynamics of the following parameters were monitored: blood pressure, heart rate, ECG alterations, myocardial damage markers - creatine phosphokinase, $\mathrm{MB}$ - creatine phosphokinase and troponin fraction, full blood count, biochemical indicators: hepatic (GGT, ASAT, ALAT, total and direct bilirubin, alkaline phosphatase) and renal (urea, creatinine).

\section{RESULTS AND DISCUSSION}

1. Demographic Characteristics and CoMorbidity in Acute Exogenous Cardiotoxic Drug Intoxications

Age: Patients with self-poisoning with high doses of cardiotoxic lipophilic drugs treated with LE at the Clinic of Toxicology at the Naval Hospital-Var- 
na in the period 2016-2018 were 16 to 84 years old. For the period 2016-2018, the largest share was that of patients between $45-60$ years (30.76\%), and the smallest was that of individuals 24 years of age or less (19.21\%). The proportion of self-poisoning predominates among the working population. For 2016, the average age was 34.83 years, for 2017 - 51.77, and for 2018 - 47.81. The highest was the average age in 2017, and the lowest was in 2016. The ratio of women to men was 12:1. Two suicides were registered for persons under 18 years.

Comorbidity: Depression was the most common disease in the analyzed population (44.11\%), followed by arterial hypertension (11.76\%), bipolar affective disorder (11.76\%), schizophrenia (8.82\%), atrial fibrillation (5.88\%), heart failure (5.88\%), dietary disorders, such as anorexia and cachexia (5.88\%), diabetes mellitus (2.94\%), and malignancies (2.94\%).

\section{Etiological Distribution of Acute Exogenous} Intoxications with Cardiotoxic Lipophilic Drugs

Frequently chosen medications used for selfpoisoning are antidepressants (30.9\%), followed by neuroleptics $(21.81 \%)$, cardiovascular medications (18.18\%), benzodiazepines (10.9\%), mixed with alcohol (5.45\%), others (5.45\%) and anxiolytics (1.81\%). Two patients had a second suicide attempt using drugs. Antidepressants occupy the major part of acute exogenous intoxications. The most commonly used drug was Deanxit (31.25\%), followed by trazodone (18.75\%), selective serotonin reuptake inhibitors (SSRIs) (31.25\%), serotonin and norepinephrine reuptake inhibitors (SNRIs) (12.5\%), and tricyclic antidepressants (TCAs) (6.25\%).

\section{Cardiotoxicity}

To evaluate cardiotoxicity, we assessed changes in blood pressure, rhythm and conduction disorders, and the presence of exotoxic shock. The following changes, not related to a previous disease, were recorded: arterial hypotension (36.4\%), followed by arterial hypertension (21.2\%), sinus bradycardia (12.1\%), sinus tachycardia (9.09\%), prolonged QT interval (6.06\%), exotoxic shock, and complete atrioventricular (AV) block (3.03\%).

\subsection{Prolonged QT Interval}

We observed two cases (5.71\%) of prolongation of the QT interval on arrival at the Clinic of Toxicology at the Naval Hospital-Varna. One was Lexotan intoxication and one - haloperidol intoxication. Fig. 1 shows prolonged QT interval $(>0.48$ at heart rate of 55 beats per minute (bpm)) as a result of Lexotan poisoning. Fig. 2 shows a normalized sinus rhythm one hour after application of Intralipid 20\% $500 \mathrm{~mL}$ bank for 24 hours $(20 \mathrm{~mL} / \mathrm{h})$. Normalized QT interval: 0.44 at heart rate of $61 \mathrm{bpm}$. The patient's pulse rose from $55 \mathrm{bpm}$ to $61 \mathrm{bpm}$.

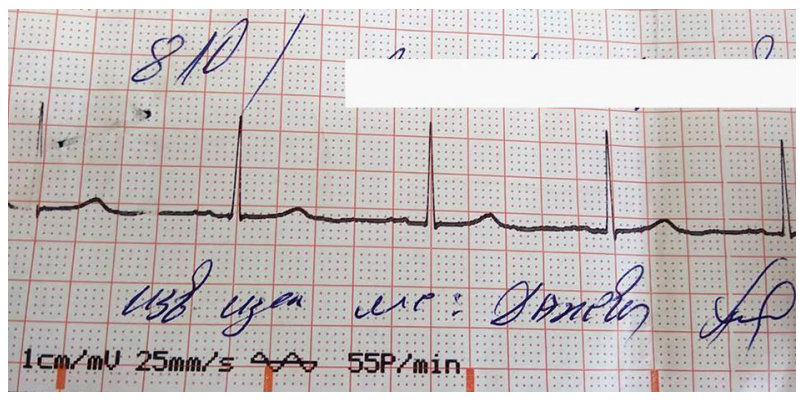

Fig. 1. Prolonged QT interval after acute exogenous intoxication with Lexotan

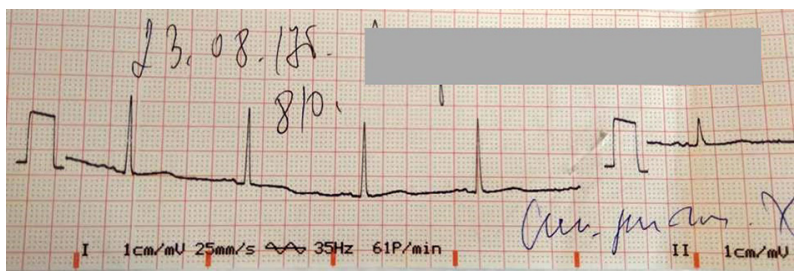

Fig. 2. Renewed sinus rhythm after application of $20 \% \mathrm{LE}$

\subsection{AV Block}

A complete AV block was observed in one of our patients (2.85\%), intoxicated with verapamil and digoxin. Fig. 3 shows the complete AV block with a heart rate of $44 \mathrm{bpm}$ and a pronounced ST depres-

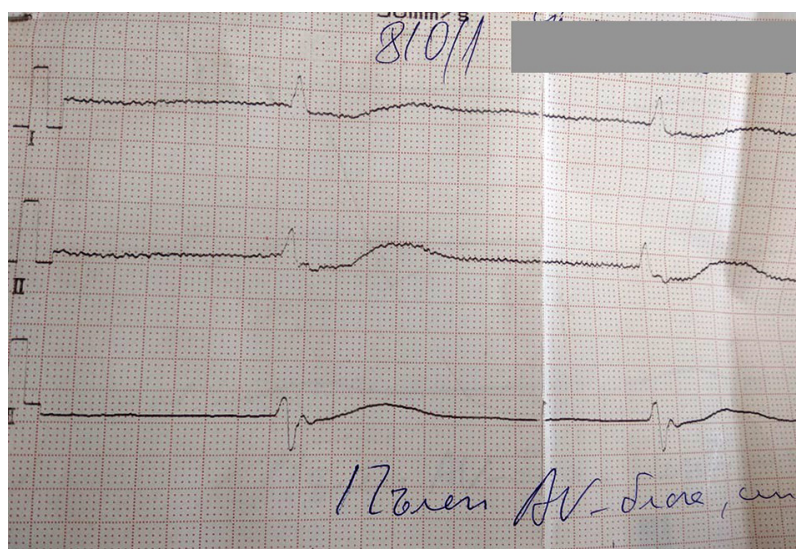

Fig. 3. Complete AV block after acute intoxication with verapamil and digoxin 
sion. Fig. 4 shows a well-documented reversal of a full AV block one hour after Lipovenoes 10\% $500 \mathrm{~mL}$ for 24 hours $(20 \mathrm{~mL} / \mathrm{h})$. This patient received a total of $4 \mathrm{LE}$ banks for 3 days to stabilize hemodynamics, resolving repolarizing ECG changes and controlling cerebrotoxic syndrome.

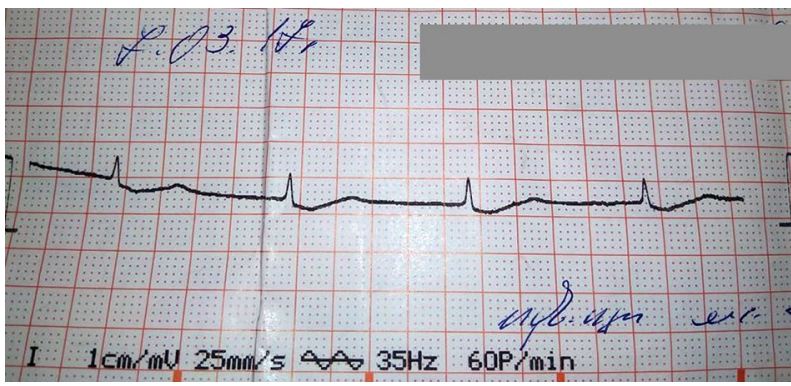

Fig. 4. AV block $2^{\text {nd }}$ degree, caused by verapamil and digoxin overdose, after Lipovenoes $10 \%$

\subsection{Bradycardia}

We demonstrate a case of bradycardia with combined poisoning with verapamil and Digoxin and controlling it after Lipovenoes $10 \% 500 \mathrm{~mL}$ for 24 hours. Fig. 5 shows the ECG-registered bradycardia in acute intoxication with verapamil and digoxin. Fig. 6 shows the ECG changes one hour after Lipovenoes $10 \% 500 \mathrm{~mL}$ administration for 24 hours. The severity of bradycardia has decreased, the pulse of the patient has increased from 44 to $56 \mathrm{bpm}$.

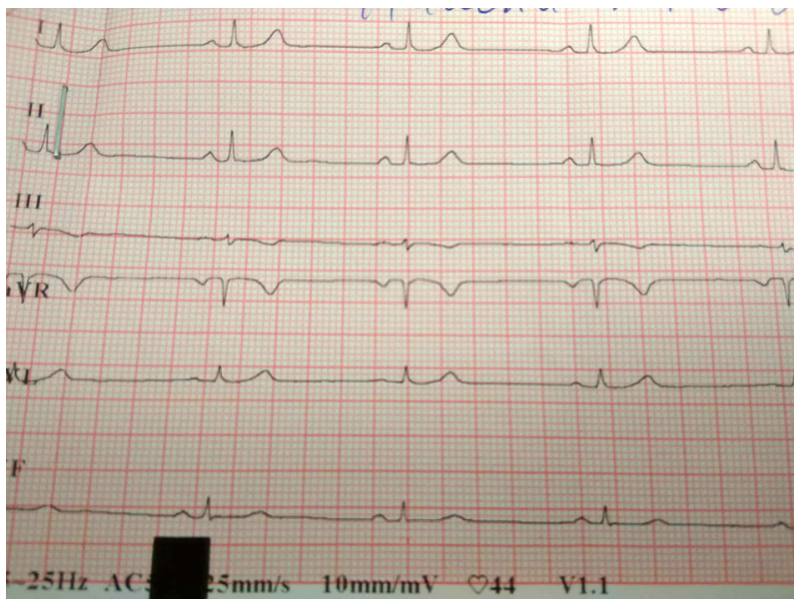

Fig. 5. Bradycardia after acute intoxication with verapamil and digoxin (heart rate 44 beats per minute)

\subsection{Tachycardia}

We demonstrate a good response to tachycardia from an overdose of serotonin antidepressant

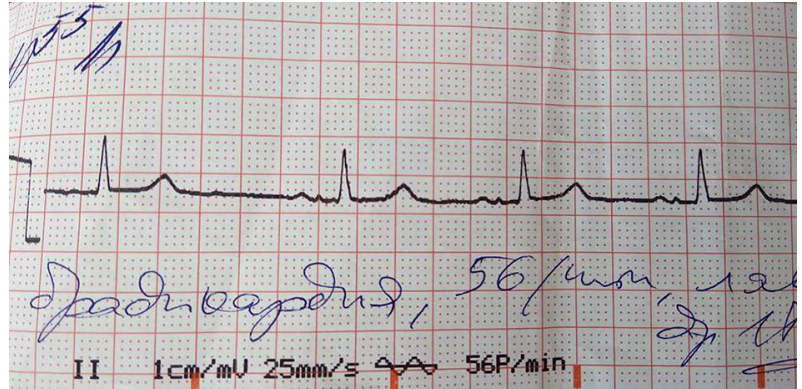

Fig. 6. Reduced bradycardia, caused by verapamil and digoxin one hour after Lipovenoes $10 \%$ (heart rate 56 beats permin.)

one hour after administration of a slow infusion of Periolimel $2000 \mathrm{~mL}$ for 48 hours $(40 \mathrm{~mL} / \mathrm{h}$ ). Fig. 7 shows ECG-registered tachycardia caused by antidepressant SNRI-venlafaxine poisoning: heart rate 117 bpm. Fig. 8 shows a reversal of the tachycardia, 24 hours after intravenous Periolimel administration. Heart rate is $79 \mathrm{bpm}$.

\subsection{Atrial Fibrillation}

We demonstrate a delay in absolute arrhythmia after administration of LE. Fig. 9 shows the registered on ECG absolute arrhythmia of atrial fibrillation, caused by intoxication with haloperidol. The heart rate decreased 1 hour after Intralipid 20\%

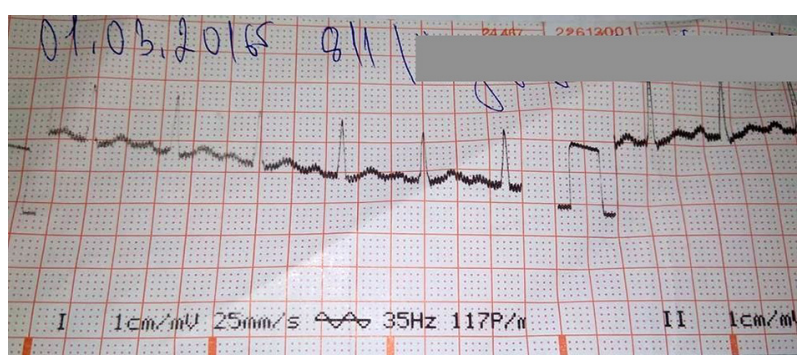

Fig. 7. Tachycardia, caused by SNRI (heart rate 117 beats per minute)

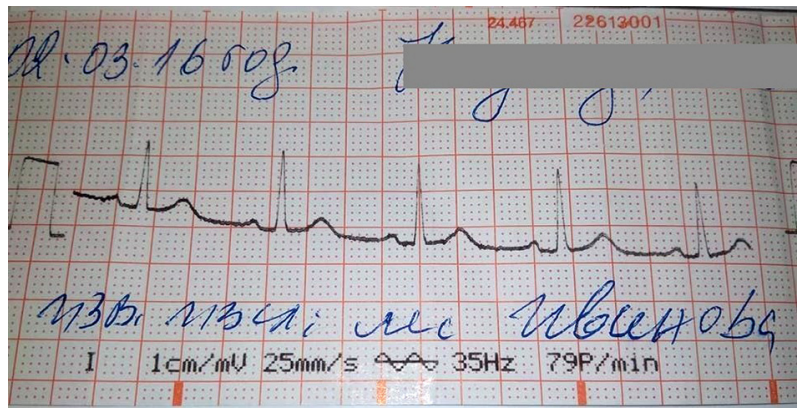

Fig. 8. Reversal of tachycardia, induced by SNRI 24 hours after Periolimel administration (heart rate 79 beats per min.) 
Gabriela Kehayova, Snezha Zlateva, Petko Marinov

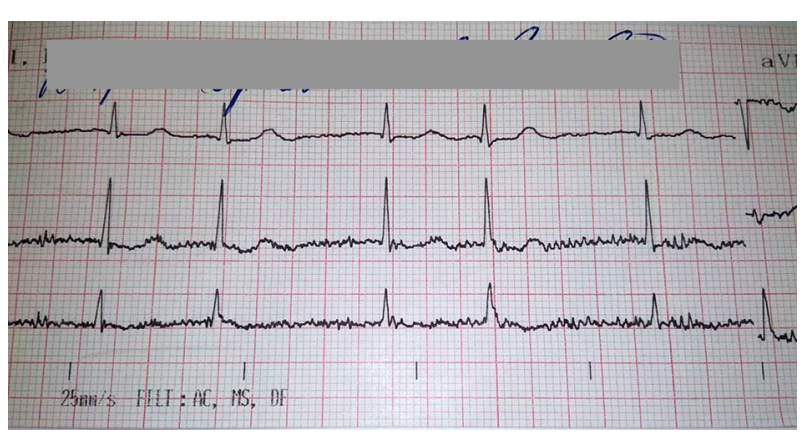

Fig. 9. Absolute arrhythmia at atrial fibrillation, $f$-waves, induced by Haloperidol overdose

$500 \mathrm{~mL}$ for 24 hours, but continued to persist due to the chronic heart disease of the patient.

\subsection{Blood Pressure}

Changes in blood pressure in acute exogenous intoxications may be in the direction of decrease or increase.

3.6.1. Arterial hypertension was observed in 7 patients (20\%), ranked by the incidence of intoxications: intoxicated with neuroleptics (four), with antiepileptic drugs (one) and with Deanxit (two).

3.6.2. Arterial hypotension was observed in $14(40 \%)$ of the patients, ranked by the incidence of intoxications: verapamil, digoxin, chlorprothixene, amitriptyline, combined with wine, clonidine, benzodiazepines, valproic acid, quetiapine, and nebivolol. In these cases various forms of LEs have been administered. Periolimel was administered in two patients, Intralipid $20 \%$ in 7, Lipovenoes $10 \%$ in 4 and Cabiven $20 \%$ in one. LEs were administered slowly intravenously for 12 or 24 hours, $40 \mathrm{~mL} / \mathrm{h}$ and 20 $\mathrm{mL} / \mathrm{h}$, respectively. LE rapidly increased blood pressure in these patients during the first 30 minutes. In cases of minor intoxications, especially in young patients, arterial pressure remained normal and no rhythmical and conduction disturbances were observed.

3.6.3. Exotoxic shock and coma were observed in one patient receiving an overdose of benzodiazepines. Simultaneously with a dopamine infusion, Intralipid 20\% $500 \mathrm{~mL}$ was administered for 2 hours $(250 \mathrm{~mL} / \mathrm{h})$ followed by another $500 \mathrm{~mL}$ for 24 hours or $(40 \mathrm{ml} / \mathrm{h})$. Within 4 hours the exotoxic shock was overcome and the woman came out of the comatose state after 36 hours.
Laboratory Indicators in Acute Exogenous Intoxications with Cardiotoxic Drugs

Patients with hypoxemia (52\%) were predominant, followed by metabolic acidosis (20\%), hyperglycemia (16\%) and elevated cardiac enzymes - CPK, $\mathrm{MB}$ creatine phosphokinase (TnI, TnT) - 12\%.

\section{Treatment Course}

The therapy at the Clinic of Toxicology at the Naval Hospital-Varna resulted in a complete cure in 25 of the patients $(96.2 \%)$ and 1 case with a lethal outcome $(3.85 \%)$ due to complications during the course of the treatment, namely pneumonia, acute respiratory failure and exotoxic shock. The average length of stay was 2.88 days. Standard detoxification therapy including gastric lavage or lavage with activated charcoal, infusions of fluid, electrolyte and glucose solutions, nootropic agents, Group B vitamins, and oxygen was performed in all patients. One patient was intubated and ventilated for 24 hours. For correction of metabolic acidosis, sodium bicarbonate was administered in bradycardia - atropine; and dopamine in a cardiogenic shock.

\section{Monitoring the Effects of LEs}

In 26 patients with acute drug poisoning with pronounced cardiotoxic effects, LE was administered on their admission into the Clinic of Toxicology at the Naval Hospital-Varna. LE was administered as continuous intravenous infusion for 12-24 hours, 20$40 \mathrm{~mL} / \mathrm{h}$, respectively. In all, hemodynamic stabilization was observed in the first hours, and the exotoxic shock (together with dopamine infusion) was controlled. Normalization of sinusoidal deviations, repolarization changes and QT interval were recorded. During treatment with LE, no significant side effects from LE were observed in any of the 26 patients. Side effects were monitored for liver, kidney, and platelets. The mean aspartate aminotransferase was $30.29 \mathrm{U} / \mathrm{L}$, alanine aminotransferase $18.43 \mathrm{U} / \mathrm{L}$, gamma glutamyltranspeptidase $31.38 \mathrm{U} / \mathrm{L}$, creatinine $65.34 \mu \mathrm{mol} / \mathrm{L}$, urea $4.43 \mathrm{~mol} / \mathrm{L}$, total bilirubin $8.83 \mu \mathrm{mol} / \mathrm{L}$, platelets $254.5 \mathrm{~g} / \mathrm{L}$. Liver, renal and platelet counts remained in the norm in all patients observed.

\section{CONCLUSION}

LE was administered to 26 patients at an average age of 44.8 years, with women prevailing (92.3\%). The ratio of women to men was 12:1. All intoxications were suicidal. The prevalence of poisoning 
Lipid Emulsions in the Treatment of Acute Poisoning in the Clinic of Toxicology at the Naval Hospital-Varna, Military Medical Academy-Sofia

with antidepressants predominated (30.9\%), followed by neuroleptics (21.81\%), cardiovascular drugs (18.18\%), benzodiazepines (10.9\%), anticonvulsants (5.45\%), combined with alcohol (5.45\%), and anxiolytics (1.81\%). Cardiotoxic changes included arterial hypotension (36.4\%), followed by arterial hypertension $(21.2 \%)$, sinus bradycardia (12.1\%), sinus tachycardia $(9.09 \%)$, absolute arrhythmia in atrial fibrillation (9.09\%), prolonged QT interval (6.06\%), exotoxic shock (2.85\%), and complete AV block (3.03\%). We have not registered complications following the administration of $20 \%$ LE, which confirms its safety in therapeutic doses. The good clinical results we have come to, rise to expectations in the scientific community that this method should be implemented as an antidote for a number of lipophilic drug intoxications.

\section{REFERENCES}

1. Kayipmaz AE, Kavalci C, Bayraktar N, Bacanli D, Aydos TR, Giray TA, et al. Treatment with lipid emulsion decreases high levels of phenytoin in rats. Biomed Res. 2017; 28 (Special Issue): S25-9.

2. Zyoud SH, Waring WS, Al-Jabi SW, Sweileh WM, Rahhal B, Awang R. Intravenous lipid emulsion as an antidote for the treatment of acute poisoning: $A$ bibliometric analysis of human and animal studies. Basic Clin Pharmacol Toxicol. 2016;119(5):512-519. doi: 10.1111/bcpt.12609.

3. Weinberg GL, VadeBoncouer T, Ramaraju GA, Garcia-Amaro MF, Cwik MJ. Pretreatment or resuscitation with a lipid infusion shifts the dose-response to bupivacaine-induced asystole in rats. Anesthesiology. 1998;88(4):1071-5. doi: 10.1097/00000542-199804000-00028.
4. Huang JM, Xian H, Bacaner M. Long-chain fatty acids activate calcium channels in ventricular myocytes. Proc Natl Acad Sci USA. 1992;89(14):6452-6. doi: 10.1073/pnas.89.14.6452.

5. Sirianni AJ, Osterhoudt KC, Calello DP, Muller AA, Waterhouse MR, Goodkin MB, et al. Use of lipid emulsion in the resuscitation of a patient with prolonged cardiovascular collapse after overdose of bupropion and lamotrigine. Ann Emerg Med. 2008;51(4):412-5. doi: 10.1016/j. annemergmed.2007.06.004.

6. Paskaleva I, Schmilev T. For some critical conditions in the course of acute poisoning in children. National Conference on Toxicology, Sunny Beach, September 2017.

7. Marinov P, Ivanov D, Zlateva S, Dimitrova S, Kehayova G, Radeva M, et al. Intravenous lipid emulsion in acute intoxication with fenitrothion. Scr Sci Pharmaceutica. 2018;5(1):47-50. doi: 10.14748/ssp. v1i1.5108.

8. Marinov P, Ivanov D, Zlateva S, Dimitrova S, Kehayova G, Radeva M. Intravenous lipid infusion in toxicological practice. Scr Sci Pharmaceutica. 2018;5(1):40-6. doi: 10.14748/ssp.vli1.5047.

9. Marinov P. Acute intoxications - contemporary look. Varna: Steno; 2018. p.91.

10. Zlateva S. Marine Toxicology. Poisoning with food from the sea and incidents with marine animal. Varna: Steno;2018. p. 66, 74. 